\title{
COMPARISON OF OPEN-FIELD, ANECHOIC CHAMBER AND TEM CELL FACILITIES/TECHNIQUES FOR PERFORMING ELECTROMAGNETIC RADIATED EMISSIONS MEASUREMENTS
}

\author{
M. L. Crawford
}

Electromagnetic Fields Division

National Bureau of Standards

Boulder, Colorado 80303

\section{ABSTRACT}

This paper compares the results of measurements performed at discrete frequencies between 30 MHz to 300 MHz using a spherical dipole reference standard radiator to evaluate: 1) a $6.0 \mathrm{~m} \times 9.0 \mathrm{~m}$ ground screen open-field site, 2) a $3.0 \mathrm{~m} \times 4.88 \mathrm{~m}$ $x 6.1 \mathrm{~m}$ anechoically quieted shielded enclosure, and 3) a $2.8 \mathrm{~m} \times 2.8 \mathrm{~m} \times 5.6 \mathrm{~m}$ anechoically quieted TEM cell for use in measuring radiated rf emissions from electronic equipment. The paper briefly describes each facility, the test techniques, and the test configuration used. The results given provide a comparison of the radiated measurement uncertainty anticipated from a point source radiator for each facility, when all known correction factors (assuming far-field conditions) are applied.

\subsection{INTRODUCTION}

New regulations require the measurement and compliance within specified limits of radiated emissions from electronic equipment [1]. These measurements are recommended to be performed on $3 \mathrm{~m}, 10 \mathrm{~m}$, or $30 \mathrm{~m}$ open-field sites, preferably with metal ground planes. The use of such facilities, however, have a number of well known limitations, such as site availability, exposure to high rf background environment, and exposure to weather. These limitations have led to the suggested use of indoor laboratory, shielded enclosures. Two important options are 1) anechoically quieted shielded rooms and 2) transverse electromagnetic (TEM) cells.

This paper summarizes the results of measurements made to evaluate and compare an anechoically quieted, shielded enclosure and a large TEM cell with a $3 \mathrm{~m}$ open-field site as alternative facilities to measure radiated emisssions from electronic equipments. The three specific facilities evaluated and the associated test techniques are described in sections 2,3 , and 4 with sufficient theory necessary for comparing the test results to free-space conditions. A summary of the measurement results and conclusions are then given in sections 5 and 6 .
The measurements were made using a $10 \mathrm{~cm}$ diameter, spherical dipole to generate reference standard fields [2] in the three different facilities.

\subsection{OPEN-FIELD SITE MEASUREMENTS}

The equivalent free-space field radiated by the spherical dipole radiator can be obtained from open-field site measurements as follows:

A. The radiated field from the dipole is measured at the desired far-field distance, using a calibrated antenna and receiver. This field can be expressed as:

$$
\left|E_{m}\right|=(A F)\left|V_{0}\right|
$$

at a prescribed far-field distance where:

$A F$ is the receiving antenna factor, and

$\left|v_{0}\right|$ is the rf voltage measured by the cal ibrated receiver.

B. To relate the field, $E_{m}$, to the equivalent free-space or direct path field, $E_{d}$, the effect of ground reflection must be accounted for. Referring to figure 1 , the magnitude* of $E_{d}$, at a distance $d$ can be determined from the measured field, $E_{m}$, using the equation [3]

$$
\left|E_{d}\right|=\frac{\left|E_{m}\right|}{\sqrt{1+B^{2}+2 B \cos \alpha}},
$$

where $B=\rho \frac{d}{r}, \alpha=\frac{2 \pi(r-d)}{\lambda}+\psi, \rho$ and $\psi$ are the magnitude and phase of the ground screen's reflection coefficient, $\lambda$ is wave length in meters, and the parameters $d$ and $r$ are shown in figure 1. Expression (2) assumes far-field conditions and is valid

\footnotetext{
*The determination of $E_{d}$ is based upon amplitude only measurements, hence, only the magnitude is obtained.
} 
for horizontal polarization, assuming dipole emitter and receiving patterns.

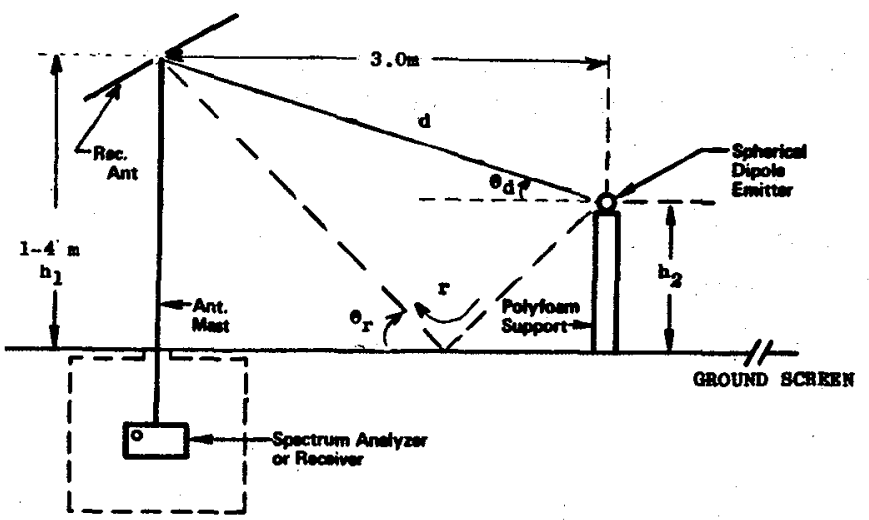

Figure 1. Configuration for measuring and calculating emissions from NBS spherical dipole on $6 \mathrm{~m} \times 9 \mathrm{~m}$ ground screen openfield site.

The receiving dipole's impedance is influenced by the proximity effect of the ground screen which, in turn, will influence the dipole's AF [4]. For half-wave dipoles, this effect can be corrected for using the graph of figure 2 , where $E_{m}$ is the measured electric field amplitude as defined for eq (1) and $E_{t}$ is the true-electric field amplitude. It is important to note that if the receiving dipole antenna is calibrated at the same height above the ground screen as its intended use, the proximity effect of the ground plane is included in the AF.

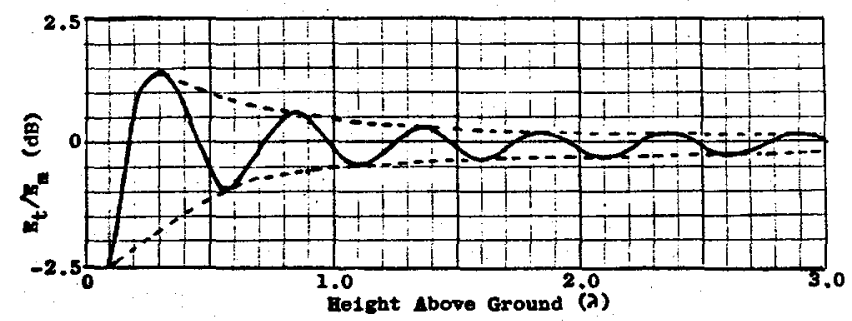

Figure 2. Ratio of measured electric field, $E_{m}$, to true field $E_{t}$, to correct for proximity effect of metal ground screen on halfwave receiving dipole's impedance.

Another correction, which may be useful to apply to the open-field site measurements, is to normalize the results to a fixed separation distance between the source and the receiving antenna. (For example, curves $B$ and $C$ of figure 7 were normalized to correct the results to a fixed separation distance of $3.0 \mathrm{~m}$ for all receiving antenna heights.)

A diagram of the $3.0 \mathrm{~m}$ open-field site measuring system showing the placement location of the spherical dipole radiator and receiving antenna is shown in figure 3. The floor of the $6.0 \mathrm{~m} \times 9.0 \mathrm{~m}$ platform was covered with 1/4-inch galvanized wire mesh which serves as a reference ground plane. A dielectric support tower constructed from fiberglass and wood was used to vary the height of the receiving dipole antenna. The tower is equipped with a remotely controlled, motor driven, dielectric chain assembly which is used to raise or lower the antenna from $1.0 \mathrm{~m}$ to $4.0 \mathrm{~m}$ as required for the measurements. The measurement plane of the antenna is located $3.0 \mathrm{~m}$ from a dielectric support platform used to mount the spherical dipole radiator on top a rotatable wood platform. This rotating platform is manual1y operated for a complete $360^{\circ}$ rotation.

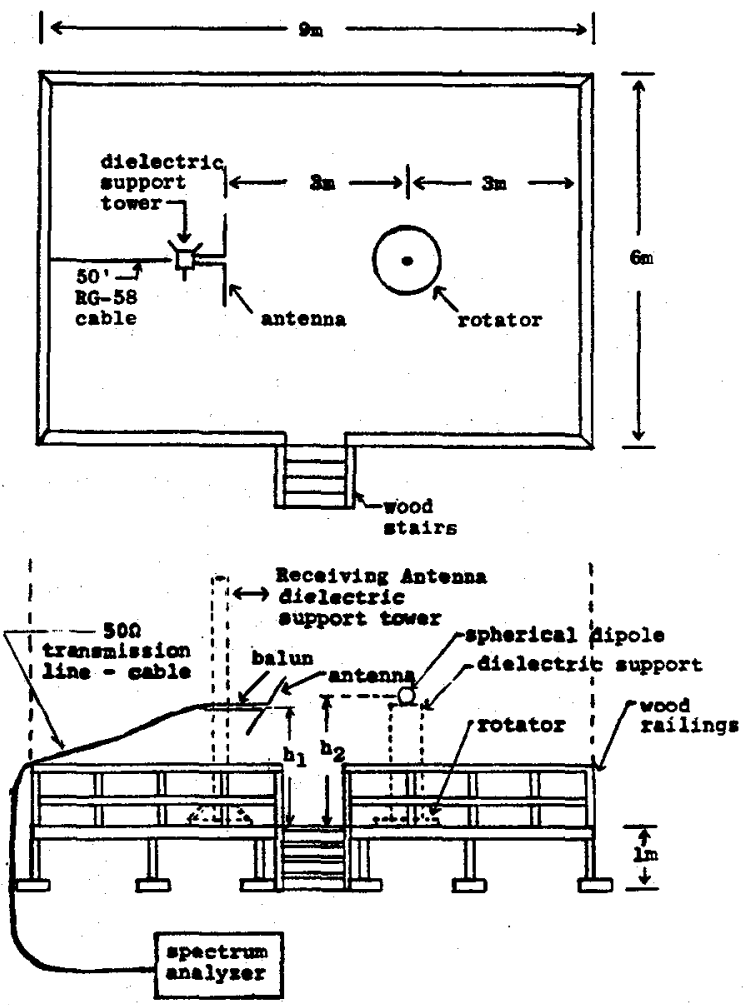

Figure 3. Diagram of $3 \mathrm{~m}$ ground screen open-field site showing placement location of spherical dipole radiator and receiving antenna. $h_{1}=1-4 \mathrm{~m}, h_{2}=0.76 \mathrm{~m}$ and $2 \mathrm{~m}$.

\subsection{ANECHOICALLY QUIETED ENCLOSURE MEASUREMENTS}

Anechoic enclosures are used to provide a simulation of an electrically quiet free-space environment. For such measurements, the signal incident upon the receiving antenna is assumed to be exclusively the direct-path signal. The amplitude of the electric field, assuming a far-field separation distance, is then obtained from the expression:

$$
\left|E_{d}\right|=(A F)\left|V_{0}\right|
$$

Diagrams of the anechoically quieted enclosure are shown in figure 4 . This figure gives the placement locations for the spherical dipole radiators and receiving antennas for the test scans. The position of the radiator was fixed, at two 
different heights, and the receiving antenna height was varied from $1.0 \mathrm{~m}$ to $2.4 \mathrm{~m}$ (figure 4 ).

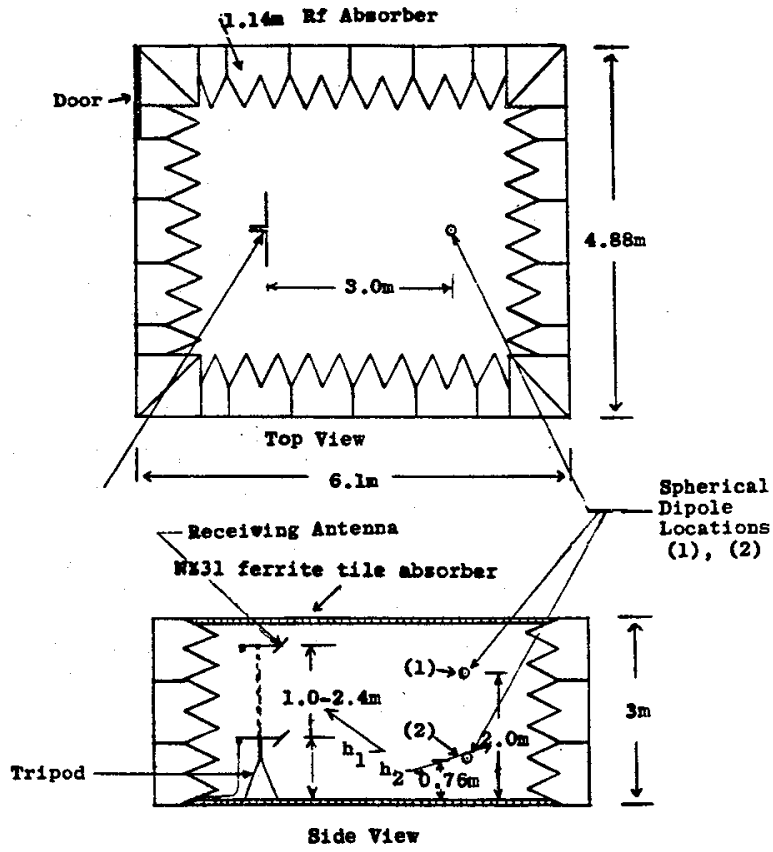

Figure 4. Anechoically quieted shielded enclosure showing placement of spherical dipole radiator and receiving antenna for vertical scan, 1 - $2.4 \mathrm{~m}$ with receiving antenna. Spherical dipole placed at location 1 or 2 for scans.

The measurement system is essentially the same as that used for the open-field site measurements with the exception of a different length transmission cable between the receiving antenna and the spectrum analyzer. This difference in cable length was accounted for in determining the antenna factor for the system.

\subsection{TEM CELL MEASUREMENTS}

The detailed procedures for performing radiated emissions measurement using a TEM cell are contained in [5]. Basically, this consists of placing the equipment under test (EUT) inside the cell in the desired orientation and test configuration, and then using the cell as a transducer to detect emissions from the operating EUT. Energy emitted from the EUT is coupled via the TEM mode of the cell to the cell's terminals where it is measured by a calibrated receiver. The magnitude of the equivalent free-space radiated field, $E_{d}$, at a distance, $d$, from the EUT is then given as

$$
E_{d}=\frac{b \eta_{0} V_{m}}{d Z_{0} K(I) \lambda_{0} \tilde{E} \cos \theta} \sqrt{\frac{2 G}{3}}
$$

where $\eta_{0}$ and $Z_{0}$ are theoretical values assumed under perfect conditions, or $\eta_{0} \cong 377 \Omega$ and $z_{0} \cong$ $50 \Omega$; b is the separation distance between the cell's center plate and lower (or upper) wall, $V_{m}$ is the voltage measured at one terminal of the cell with the opposite terminal, terminated into 502; $K(I)$ is the change in the EUT radiation current caused by enclosing the EUT inside the confining environment of the cell. (If the EUT is small, $K(I)$ is assumed to be 1 ); $\lambda_{0}$ is the wavelength of the radiated signal in meters; $E$ is the normalized electric field inside the cell relative to the field strength at the center of the cell test region; $\cos \theta$ corresponds to the polarization match between the radiated field from the EUT and the TEM mode field characteristics of the cell; and $G$ is the gain of the EUT as a radiator. Note that eq (4) is valid only for a symmetrically designed TEM cell.

A number of precautions must be observed when using a TEM cell to insure repeatable, meaningful EMC measurement results. These precautions are described in [5] and [6].

A new approach has recently been developed at NBS (i.e., since obtaining the data contained in this paper) which uses a TEM cell to more completely quantify radiated emissions from EUT $[7,8]$. This approach, for some limited applications, will provide the detailed free-space radiation pattern and total radiated power from an EUT.

A complete physical description of the $2.8 \mathrm{~m} \times$ $2.8 \mathrm{~m} \times 5.6 \mathrm{~m}$ TEM cell used in this comparison study is given in [9]. Cross-sectional views of the cell showing placement locations of the spherical dipole radiator are shown in figure 5 . Measurements were made with the radiator placed at eleven different locations to evaluate the cell's coupling characteristic for measuring the radiator's emissions.

The block diagram of the TEM cell measurement system is shown in figure 6 . Plastic foam was used to support the spherical dipole radiator inside the cell.

\subsection{MEASUREMENT RESULTS}

Measurements were made of the horizontally polarized, radiated field strength from the spherical dipole radiator as per the position diagrams of figure 3. Horizontally polarized resonant dipoles were used as the receiving antennas. These results are shown in figure 7 . Curves $A$ give the measurement results converted to field strength using expression (1). Curves $B$ were obtained using eq (2) corrected for proximity effects of the ground screen on the receiving antenna's impedance and is the field strength due to the direct-path signal contribution (i.e., the equivalent freespace, far-field radiated signal) normalized for a source-to-receive antenna separation distance of $3.0 \mathrm{~m}$. 
Curves $c$ are the field-strength measurement results obtained using expression (3), as per the position diagram of figure 5, inside the anechoically quieted enclosure. Again, the results were normalized for a source-to-receiving antenna separation distance of $3.0 \mathrm{~m}$. Some of the uncertainties present at frequencies below approximately $90 \mathrm{MHz}$ are due to insufficient measurement separation distances (i.e., measurements were assumed to have been made in the far field, which is not true at the lower frequencies). Note that curves $B$ compare to curves $C$ within $7 \mathrm{~dB}$.

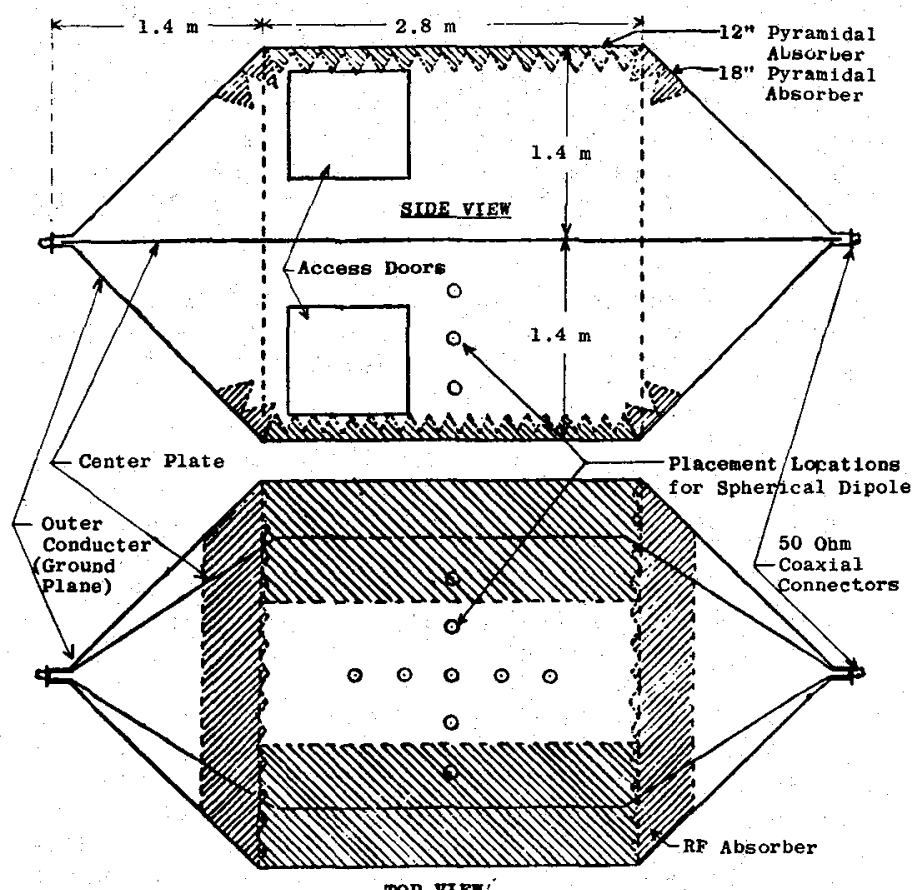

Figure 5. Diagram for absorber loading TEM cell. (Shaded areas indicate placement of anechoic material.) Test locations for placement of spherial dipole radiator al so shown.

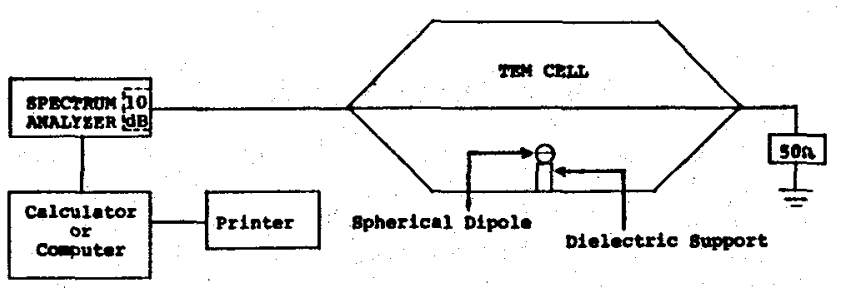

Figure 6. Block diagram of system for measuring radiated emissions from spherical dipole placed inside TEM cell.

Results of the measurements made to determine the equivalent free-space radiated field strength $3 \mathrm{~m}$ from the spherical dipoles using the TEM cell are summarized in figure 8 . These results were obtained (as per the block diagram of figure 6 and spherical dipole position diagram of figure 5) by measuring the signal radiated from the dipole placed at the various locations inside the TEM cel1. Energy radiated from the dipole is coupled via the TEM mode of the cell to the cell's output port where it was measured using a spectrum analyzer. Equation (4) was then used to calculate the equivalent free-space radiated field. Data above the dashed line indicate the frequencies for which the cell operates with only its fundamental TEM mode. These measurements should be accurate to within $\pm 2 \mathrm{~dB}$. [2]. The data shown at $90 \mathrm{MHz}$ will have somewhat less accuracy due to the potential resence of the $T E_{01}, T E_{10}$, and $T E_{11}$ modes in the 211. At frequencies above approximately $110 \mathrm{MHz}$, oth TE and TM waveguide modes can exist which zuse significant degradation in the measurement zcuracy [10]. An examination of figure 8 data ives some indication of the precision of the sasurements as a function of EUT placement cation and frequency. For example, at $120 \mathrm{MHz}$, 10 standard deviations from the mean value of the sasured data are $4.32 \mathrm{~dB}$. This means that $95 \%$ of le time the radiated emissions from the spherical pole should be within $\pm 4.32 \mathrm{~dB}$ of the mean value lown.

\subsection{CONCLUSIONS}

Obtaining repeatable, meaningful results of idiated emissions becomes progressively harder for Iccessively more complex EUT's, thus increasing ie measurement uncertainties. The intent of the irk described in this paper was to compare gdiated emission measurement results obtained sing different facilities/techniques; hence a well zfined, point source radiator was used as a :ference standard EUT. The results, though imewhat limited in scope, should give insight into ie feasibility and/or desirability of using a irticular type of facility for measuring radiated emissions from EUT as a function of frequency.

The accuracy of the results given is limited by how well the radiation characteristics of the reference standard spherical dipole radiator are known, and by how accurate the receiving antenna and measurement instrumentation characteristics are known. The $10 \mathrm{~cm}$ diameter spherical dipole has well-defined radiation characteristics and can be used to establish known reference fields within an estimated $\pm 3 \mathrm{~dB}$ at the discrete frequencies indicated in this paper. A number of conclusions can be made from the limited data obtained in this study. Some are listed below:

(a) The open-field measured results, when corrected for reflections from the ground screen, varied up to $5 \mathrm{~dB}$ from the calculated theoretical field strengths. This was due to the limited measurement accuracy and dynamic range of the measurement system, the departure from the assumed perfect reflectivity of the ground screen, and the variation from theoretically predicted radiator characteristics of the spherical dipole source, multipath, etc. 
(b) Comparison of the results obtained using the corrected open-field data and the anechoically quieted enclosure data, indicate agreement to within $8 \mathrm{~dB}$ at the selected frequencies measured from $50 \mathrm{MHz}$ to $270 \mathrm{MHz}$.

c) The measured field strength in the anechoically quieted enclosure, consistently decreased (up to $9 \mathrm{~dB}$ ) as the receiving antenna approached the enclosure's ceiling. The field strength should be constant in amplitude if the measurements were performed in a perfect free-space environment.
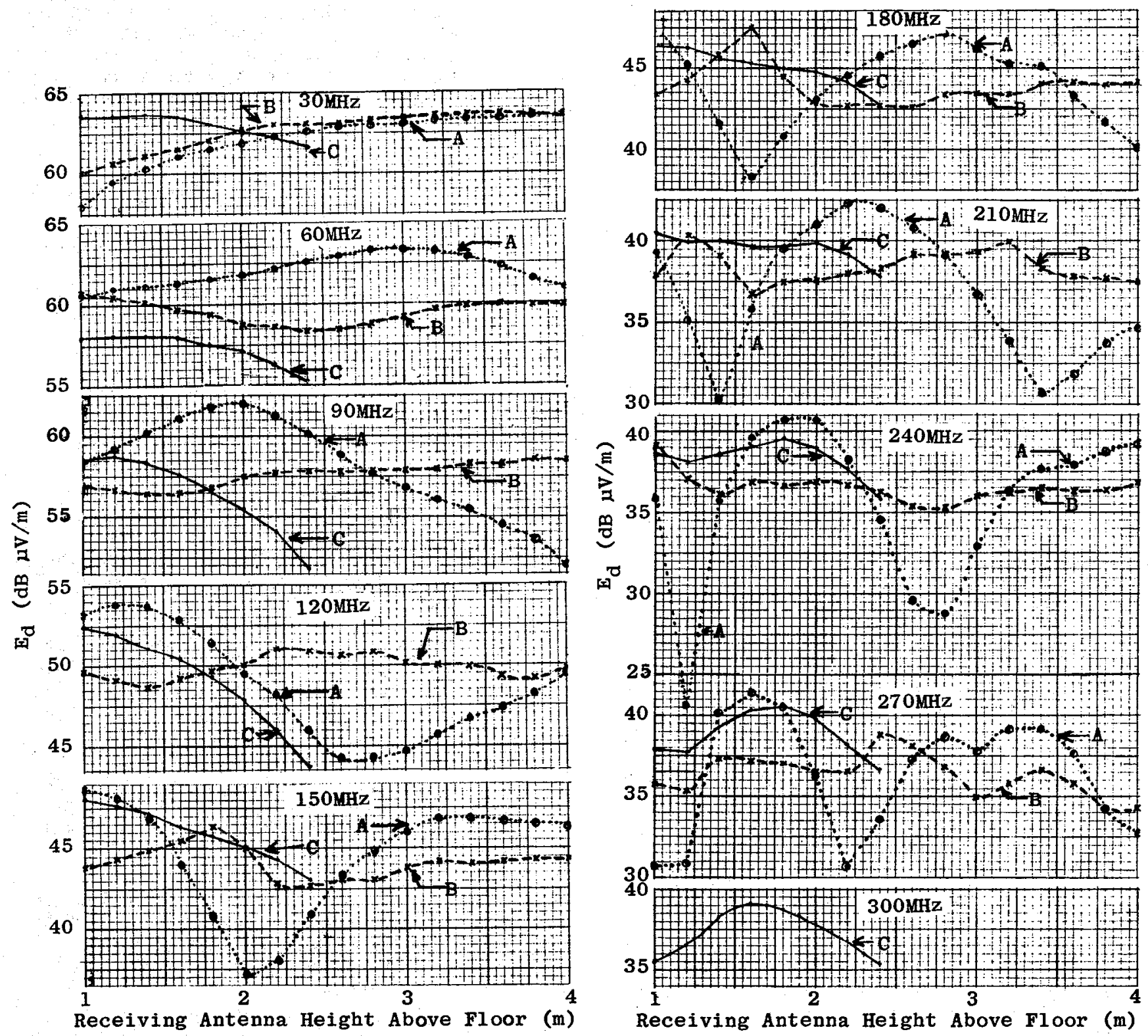

Figure 7. Electric field radiated from spherical dipole located on $3 \mathrm{~m}$ open-field site and inside anechoically quieted shielded enclosure. Curves $A$ are signal measured using resonant dipoles corrected for manufacturer's antenna factor with cable loss at the open-field site. Curves B are curves A data corrected for reflection from ground screen (open-field site), corrected for the change in antenna impedance due to the ground screen, and corrected to normalize the data to $3 \mathrm{~m}$ separation distance. Curves $C$ are for the anechoic enclosure with data normalized to $3 \mathrm{~m}$ separation distance. Source $2 \mathrm{~m}$ above floor. 
(d) Comparison of the data obtained using the TEM cell with the data obtained using the open-field site and anechoically quieted enclosure indicates agreement to within approximately $8 \mathrm{~dB}$ at $30 \mathrm{MHz}$ and $60 \mathrm{MHz}$. These frequencies are below mode resonances for the cell. At higher frequencies, larger differences (up to $12 \mathrm{~dB}$ ) exist due to cell multimoding.

These values were obtained when all known correction factors were applied (assuming far-field conditions). Some of these corrections can only be made for point sources such as the spherical dipole radiator; for large radiators such as cabinets, the exact locations of the source is not known, and therefore it is impossible to apply all the corrections used with the spherical dipole.

Some of the measurements discussed in this paper were performed in the nearfield (i.e., close proximity of the source), hence, introducing significant errors proportional to the separation distance. Additional work is needed to evaluate errors associated with using conventional antennas to perform EMC measurements in the near-field and then projecting the results to other separation distances.

E-Field at 3 meters from dipole radiator $(\mathrm{dB} \mu \mathrm{V} / \mathrm{m})$

\begin{tabular}{|c|c|c|c|c|c|c|c|c|c|c|c|c|c|}
\hline $\begin{array}{c}\text { Freq } \\
\mathrm{MHz}\end{array}$ & 1 & 2 & 3 & 4 & 5 & 6 & 7 & 8 & 9 & 10 & 11 & $\bar{x}$ & $s$ \\
\hline 30 & 64.6 & 63.8 & 64.7 & 64.8 & 64.8 & 65.1 & 64.2 & 64.6 & 65.3 & 64.7 & 63.2 & 64.5 & 0.59 \\
\hline 60 & 58.9 & 58.0 & 58.5 & 57.0 & 57.0 & 59.5 & 57.8 & 58.4 & 59.9 & 58.4 & 56.8 & 58.1 & 0.88 \\
\hline 90 & 52.1 & 53.5 & 52.1 & 54.6 & 54.6 & 54.4 & 54.2 & 55.1 & 57.1 & 54.3 & 55.5 & 54.3 & 1.43 \\
\hline 120 & 47.9 & 47.2 & 48.9 & 43.1 & 43.1 & 45.5 & 41.5 & 42.7 & 43.8 & 44.6 & 41.2 & 44.0 & 2.16 \\
\hline 150 & 39.3 & 30.3 & 34.6 & 25.9 & 37.1 & 39.8 & 36.4 & 34.0 & 34.8 & 28.0 & 38.1 & 34.4 & 4.56 \\
\hline 180 & 36.4 & 31.7 & 34.5 & 33.6 & 39.7 & 31.1 & 34.6 & 34.3 & 33.2 & 41.7 & 38.9 & 35.7 & 3.42 \\
\hline 210 & 29.5 & 36.7 & 32.2 & 38.0 & 38.0 & 29.0 & 28.8 & & 29.0 & 34.1 & 34.9 & 33.6 & 4.05 \\
\hline 240 & 39.9 & 37.3 & 39.4 & 33.3 & 37.7 & 34.7 & 35.5 & 39.5 & 35.5 & 41.8 & 40.7 & 37.8 & 2.68 \\
\hline 270 & 34.2 & 42.9 & 34.0 & 43.5 & 43.5 & & 32.6 & 43.2 & 32.7 & 30.9 & & 36.4 & 5.27 \\
\hline 300 & 44.4 & 32.4 & 42.8 & 39.3 & 39.3 & 44.2 & 43.6 & 44.1 & 43.5 & 43.7 & 39.8 & 41.5 & 3.90 \\
\hline
\end{tabular}

Figure 8. Calculated equivalent free-space (direct path) radiated field from spherical dipole radiator using TEM cell measurements for dipole placement locations indicated in figure 5 . Frequencies below dotted line (over $60 \mathrm{MHz}$ ) are above TEM cell multimode cut-off.

\subsection{REFERENCES}

[1] FCC Docket 80-284, Notice of Proposed Rule Making, "In the Matter of Amendment of Parts 2 and 15 of the Rules Relating to Verification and Methods of Measurement of Computing Devices," FCC General Docket 80-284, adopted June 11,1980 and released June 20, 1980.

[2] M. L. Crawford and J. L. Workman, "Spherical Dipole for Radiating Standard Fields." Conferences of Precision Electromagnetic Measurements, Braunschweig, Germany. IEEE 80 CH 1497-71M; 424-429; 1980 June 23-27.

[3] W. S. Bennet and H. E. Taggart, "Characterization of CISPR/VDE Far-Field EMI Test Site with Ground Screen." Third Symposium and Technical Exhibition on EMC, Rotterdam, May 1-3, 1979; pp. 507-513.

[4] F. M. Greene, "Influence of the Ground on the Calibration and Use of VHF Field-Intensity Meters," Journal of Research of NBS, Vol. 44, Feb. 1950, pp. 123-130.

[5] M. L. Crawford and J. L. Workman, "Using a TEM Cell for EMC Measurements of Electronic Equipment," NBS Technical Note 1013, revised July $1981,65 \mathrm{pp}$.
[6] M. L. Crawford, "Improving the Repeatability of EM Susceptibility Measurements of Electronic components when Using TEM Cells." SAE International Congress and Exposition, Detroit, Michigan. February 28 - March 3, 1983, Technical Paper 830607.

[7] I. Sreenivasiah, D. C. Chang, and M. T. Ma, "Characterization of Electrically Small Radiating Sources by Tests. Inside a Transmission Line Cell," NBS Technical Note 1017, Feb. 1970.

[8] G. H. Koepke and M. T. Ma, "A New Method for Determining the Emission Characterisitcs of an Unknown Interference Source," IEEE Inter. Symp. on EMC, Santa Clara, CA, Sept. 8-10, 1982 ; 82CH 1718-6 EMC.

[9] W. F. Decker, W. A. Wilson, and M. L. Crawford, "Construction of a Large Transverse Electromagnetic Ce11," NBS Technical Note 1011, Feb . 1979.

[10] J. C. Tippet, "Modal characteristics of rectangular coaxial transmission line." Thesis submitted June 1978 for Degree Doctor of Philosophy to University of Colorado, Electrical Engineering Department, Boulder, $\mathrm{CO}$. 